\title{
POSTPARTUM DEPRESSION: ASSESSMENT OF CULTURAL RISK FACTORS
}

\author{
Amina Hanif Tarar, Muhammad Mohsin Ijaz, Muhammad Ali Tarar*, Munazza Batool** \\ Government College University Lahore Pakistan, * Gazi University, Dera Ghazi Khan Pakistan, **Dera Gazi Khan Medical College, \\ Dera Gazi Khan Pakistan
}

\begin{abstract}
Objective: To explore the cultural risk factors influencing the Postpartum Depression of first-born infants' mothers.

Study Design: Correlational survey.

Place and Duration of Study: Jannat Maternity Home, Dera Ghazi Khan, Pakistan, from Jan to Nov 2019.

Methodology: A sample of 101 first-born infants' mothers with ages ranging from 18 to 26 years was selected through purposive sampling. Edinburgh Post-Partum Depression Scale and Oslo Social Support Scale were used.

Results: Results suggested significantly lower mean scores for Postpartum Depression of mothers having a firstborn baby boy $(M=5.98, S D=2.44)$ and higher for those having a girl $(M=18.33, S D=4.62)$. Multi-factorial analysis of variance indicated a significant main effect of Social Support and Family System on Postpartum Depression levels of first-born infants' mothers, explaining $44 \%$ and $11 \%$ variance respectively. Further, Post-Hoc analysis revealed higher levels of Postpartum Depression for mothers having poor Social Support $(\mathrm{M}=17.30, \mathrm{SD}=6.15)$ as compared to those having moderate $(M=9.68, S D=6.27)$ and strong Social Support $(M=6.26, S D=2.30)$. Moreover, pair wise-comparisons demonstrated higher levels of Postpartum Depression for first-born infants' mothers belonging to nuclear families $(M=17.61, S D=7.09)$ as compared to joint ones $(M=9.60, S D=5.70)$.

Conclusion: The study establishes that first-born infants' gender, degree of social support, and nature of family have a profound effect on the Postpartum Depression levels of mothers. These findings will extend the understanding of cultural risk factors influencing first-born infants' mother's mental health.
\end{abstract}

Keywords: Family system, Infant, Postpartum depression, Social support.

This is an Open Access article distributed under the terms of the Creative Commons Attribution License (http://creativecommons.org/licenses/by/4.0), which permits unrestricted use, distribution, and reproduction in any medium, provided the original work is properly cited.

\section{INTRODUCTION}

Post-partum depression (PPD) is a debilitating condition occurring within one month following childbirth and is characterized typically by sadness, hopelessness, low self-esteem, guilt, sleep and eating disturbances, inability to be comforted, exhaustion, emptiness, anhedonia, social withdrawal, low or no energy, becoming easily frustrated, feeling inadequate in taking care of the baby, impaired speech or writing, spells of anger towards others, increased anxiety or panic attacks. It is necessary to distinguish PPD from "maternity/ baby blues" which constitute transitory and mild moodiness suffered by up to $80 \%$ of the women. PPD however, differs in both intensity and duration of symptoms which may last for a year ${ }^{1}$.

Correspondence: Dr Amina Hanif Tarar, Dept of Psychology, Government College University, Lahore Pakistan

Received: 06 Oct 2020; revised received: 12 Nov 2020; accepted: 18 Nov 2020
Postpartum Depression (PPD) does not only affect the mother's physical and psychological health but it also has serious consequences for the newborn. Women with post-partum major depressive episodes show highly variable maternal attitudes ranging from disinterest, fearfulness of being alone with the infant, or over intrusiveness, that inhibits adequate infant rest. The maternal consequences of PPD include health, psychological well-being, interpersonal relationships, breast feeding, maternal bond with the infant and risky behaviors. It also has consequences for the newborn's health as anthropometry, and disturbances in sleep, motor, cognitive, language, emotional and social dev-elopment ${ }^{2}$.

Risk factors for PPD can be categorized into biological, psychological, and social ones. Among the biological risk factors, hormonal dys-regulation, thyroid problems 3 , pre-menstrual syndrome $^{4}$, and preeclampsia 5 associated with PPD. 
Also, vitamin D deficiency, low Omega 3 fatty acid consumption and lack of physical activity have been attributed to modern lifestyles ${ }^{6}$ can put one at risk for PPD. Among social psychological factors, disruption of birth plans, satisfaction with the birth experience and inadequate support from maternity providers are salient in contributing to $\mathrm{PPD}^{7}$. While the risk factors for PPD may vary greatly across cultures and ethnicities ${ }^{8}$, inquiries intospecific cultural contexts revealed the alarming situation of $28 \%$ to $63 \%$ the highest one in Asia-with up to $29 \%$ found in Pakistani samples 9 , which alludes to the fact that PPD has remained a neglected issue in Pakistani health concerns.

The variability of PPD across cultures suggest socio-cultural factors an important area to be investigated. Furthermore, while the above literature presents lack of social support from maternity providers as important risk factor, it can be argued that in a family-oriented cultural context of Pakistan, social support as a contributing factor to PPD may be further investigated. Also, given that the local culture is embedded in a patriarchal tradition showing preferences for the baby boy, the gender of the newbornmay factor be investigated as a contributing to PPD. Earlier indigenous research on PPD 10 showed PPD to vary as a function of the gender of the baby but one limitation of these project was that it did not control the birth order of the child and other important demographics relevant to PPD. The current research would investigate cultural factors as the gender of the baby as well the social support and other demographics as maternal education and socio-economic class while controlling for birth order and biological factors so that treatment strategies incorporate the cultural information for better patient outcomes. It was predicted that among mothers of first-born infants, mothers of baby girls would have a significantly higher level of PPD than mothers of baby boys. Furthermore, PPD would vary as a function of social support and there would be a significant negative correlation between PPD and social support. Moreover, significant differences were expected to determine the PPD prevalence based on family system (joint or nuclear), socio-economic status, mother's education, and occupation.

\section{METHODOLOGY}

The study employed a correlational design using a survey method to gain observations on a sample consisting of 101 firstborn infants' mothers at Jannat Maternity Home, Dera Ghazi Khan, Pakistan, from January to November, 2019. The sample was drawn with a purposive sampling technique. Mothers with biological factors as thyroid problems in mothers and congenital problems in infants, birth with a c-section, as well as mothers of second and next born infants were excluded from the study. The study was conducted from January to November, 2019. The ethical principles as informed consent, the confidentiality of the participant, providing them with knowledge of the purpose of the study, and ensuring access to any publications upon their demand were thoroughly followed. Certification of following ethical standards was approved by relevant authorities (Certificate No. DJK/JMH/11/2019). Urdu version of Edinburgh Post-Partum Depression Scale ${ }^{11}$, a highly reliable and widely used measure of PPD, comprising of 10 items was used to measure Postpartum Depression. Urdu version of Oslo social support scale (OSSS-3) ${ }^{12}$ was used to measure social support. The OSSS-3 consists of three items assessing the level of social support with scores of 3 to 8 indicating poor social support, scores from 9 to 11 reflecting moderate social support, and scores from 12 to 14 strong social support. The firstborn infants' mothers were approached on their after-delivery visit (approximately 5 to 7 days after delivery) to the gynecologist. Along with noting down demographic data as age, education, occupation, socio-economic status and family system (joint / nuclear) Edinburgh Post-Partum Scale ${ }^{11}$ and OSSS-312 were given to the mothers and taken back in their next visit. The participants were also instructed to fill in the scale alone to avoid any possible social pressures on their responses. Data were analyzed using SPSS package 23 employing statistical techniques of $\mathrm{t}$-test and multi-factorial ANOVA. 


\section{RESULTS}

A total of 101 new firstborn infants' mothers participated in the study, $50(49.5 \%)$ were mothers of newborn baby boys, and 51 (50.5\%) of newborn baby girls. The age of mothers $(\mathrm{M}=22.05$

Table-I: Differences in postpartum depression of firstborn infants' mothers regarding their baby's gender $(n=101)$.

\begin{tabular}{l|c|c|c} 
Variable & $\begin{array}{c}\text { Boys' } \\
\text { Mothers } \\
\text { Mean } \pm \text { SD }\end{array}$ & $\begin{array}{c}\text { Girl's } \\
\text { Mothers } \\
\text { Mean } \pm \text { SD }\end{array}$ & $\begin{array}{c}p \text { - } \\
\text { value }\end{array}$ \\
\hline $\begin{array}{l}\text { Postpartum } \\
\text { depression }\end{array}$ & $5.98 \pm 2.44$ & $18.33 \pm 4.62$ & $0.001^{*}$ \\
\hline${ }^{*} p<0.01$ & \multicolumn{3}{|l}{}
\end{tabular}

$\pm \mathrm{SD}=1.89$ ) varied from a minimum of 18 years to a maximum of 26 years. Twenty two (21.8\%) first born infants' mothers were from lower, 39(38.6\%) from the middle, and 40 (39.6\%) were from upper socio-economic status. Forty five $(44.6 \%)$ matriculated/intermediate, $48(47.5 \%)$ bachelors, and $8(7.9 \%)$ masters. Thirty five $(34.7 \%)$ were house gender. Mothers who have first-born baby girl had higher PPD levels (18.33 \pm 4.62$)$ as compared to mothers who have a first-born baby boy $(5.98 \pm$ 2.44). Pearson Correlation indicated a high negative correlation $(r=-0.64, p<0.001)$ between Social Support and PPD.

A multi-factorial analysis of variance (ANOVA) (table-II) indicated a statistically significant main effect of Social Support $(\mathrm{F}(2,101)=$ $14.36, p<.001, \eta \mathrm{P} 2=0.44)$ and Family System ( $\mathrm{F}(1$, $101)=4.45, p=.042, \eta \mathrm{P} 2=.04)$ on PPD of firstborn infants' mothers. Partial eta Squared indicated that social support was responsible for $44 \%$ variance and family system $11 \%$ variance in PPD of firstborn infants' mothers. The observed power of the test was very high (1.0) for Social Support and satisfactory (0.54) for Family System indicating that the test was able to detect the difference it was there.

However, Socio-economic status, Mother's education, and occupation had no statistically

Table-II: Multi-factorial ANOVA and comparisons using Post-Hoc Tukey test to explore the role of social support, family system, socio-economic status, mother's education, and occupation in postpartum depression of firstborn infants' mothers $(n=101)$.

\begin{tabular}{|c|c|c|c|c|}
\hline Parameters & Mean \pm SD & Mean \pm SD & Mean \pm SD & $p$-value \\
\hline \multirow{2}{*}{ Social Support } & Poor $(n=44)$ & Moderate $(\mathrm{n}=34)$ & Strong $(\mathrm{n}=23)$ & \\
\hline & $17.30 \pm 6.15$ & $9.68 \pm 6.27$ & $17.30 \pm 6.15$ & $0.01^{* *}$ \\
\hline \multirow{2}{*}{ Socio-economic Status } & Lower $(n=22)$ & Middle $(n=39)$ & Upper $(n=40)$ & \\
\hline & $12.14 \pm 8.41$ & $13.10 \pm 7.31$ & $11.40 \pm 6.48$ & 0.897 \\
\hline \multirow[t]{2}{*}{ Mother's Education } & $\begin{array}{c}\text { Matriculated/ } \\
\text { Intermediate }(n=45)\end{array}$ & Bachelors $(n=48)$ & Masters $(n=8)$ & \\
\hline & $11.91 \pm 7.09$ & $12.46 \pm 7.53$ & $12.50 \pm 6.78$ & 0.305 \\
\hline \multirow{2}{*}{ Mother's Occupation } & Housewives $(\mathrm{n}=22)$ & School teachers $(n=39)$ & Self-employed $(n=40)$ & \\
\hline & $12.14 \pm 8.41$ & $13.10 \pm 7.31$ & $11.40 \pm 6.48$ & 0.680 \\
\hline \multirow{2}{*}{ Family System } & \multicolumn{2}{|c|}{ Joint $(n=68)$} & Nuclear $(n=33)$ & \\
\hline & \multicolumn{2}{|c|}{$9.60 \pm 5.70$} & $17.61 \pm 7.09$ & $0.042^{*}$ \\
\hline
\end{tabular}

${ }^{*} p<0.05,{ }^{* *} p<0.01$

wives, $36(35.6 \%)$ school teachers, and 30 (29.7\%) self-employed. Sixty Eight (67.3\%) belonged to the joint while $33(32.7 \%)$ were from nuclear families. Forty four (43.6\%) reported poor, $34(33.7 \%)$ moderate, and $23(22.8 \%)$ strong social support on the OSSS-32 social support scale.

The $t$-test (table-I) indicated a statistically significant difference in means scores of PPD of first born infants' mothers based on their baby's significant main effect on PPD of firstborn infants' mothers. Interaction effects between independent variables were neither the focus here nor statistically significant. Further, post hoc analysis revealed that the firstborn infants' mothers who had poor social support experienced higher levels of PPD as compared to those who had moderate and strong social support. Additionally, the exploration by pair wise comparisons revealed 
higher PPD levels for those firstborn infants' mothers from nuclear families as compared to those belonging to the joint family.

\section{DISCUSSION}

The results of the present study supported that mothers of baby girls had a significant higher level of PPD than mothers of baby boys. Social support and family system also significantly predicted PPD. This explains the findings of previous studies ${ }^{13}$, where other known PPD risk factors may be similar across different cultures, except an infant's gender. As researches from western societies demonstrated a weak or null association between the baby's gender and mother's risk of developing PPD and studies from collectivistic cultures identified a significant relation ${ }^{13}$. This highlights the influence of familial and social discriminatory attitudes toward infants' gender on mother's PPD.

Corroborating with the other research efforts $^{14}$ social support turned out to be another factor pointing the culture and social fabric. Strong social support leading to lowest and poor Social Support to highest PPD levels delineated the precedence of social support in mediating the risk factors for PPD. These results supporting previous research suggests that intensive and negative involvement of relatives in a participant's life and lack of support from husband were strongly associated with significant PPD symptoms ${ }^{15}$. Also, previous research on rural samples establishes the importance of partner's support ${ }^{16}$; social support is also the best intervention strategy to lower PPD ${ }^{17}$. Further, imputing social support in a collectivistic society like Pakistan, the family system comes first. This may be the reason why family system was also supported and concordant to other investigations of urban samples ${ }^{18}$, signifying the role of a joint family system in lowering the risk of PPD as compared to the nuclear family system. However, a sample of rural mothers with first-borns may depict different results, considering that rustic areas are also going through familial transitioning.
Socio-economic status, mother's education and occupation were not substantiated. However, other studies had shown significant differences in SES, mother's education, and occupation ${ }^{19}$. This may be due to higher sample diversity, as they may not have theoretical restrictions such as firstborn infants' mothers only.

Like any other scientific investigation, this study also has some limitations. First, narrow sample spectrum for mothers' age limiting any significant variance on maturity basis. This may happen due to cultural differences as in western cultures first pregnancy may occur much later in their life, and contrastingly Pakistani culture encourages early marriage and pregnancy. Further efforts can strive for an extended range of mothers' age. Second, self-report measures are prone to bias, which may have influenced our findings. Third, despite measures taken to avoid any influential bias, participants were still prone to be influenced by other primacy and recency effects while filling the questionnaire at home. Future investigations can incentivize the participation to pursue mothers to fill the questionnaire during their visit. Moreover, this study was conducted on an urban sample of first-born baby mothers who are more likely to visitthe gynecologist. Representation of various portions such as rural participants may provide a comprehensive picture of the Pakistani population.

\section{CONCLUSION}

The most unique findings of this study were the birth order of the infant and family system as contributing factors of PPD. Previous researches, exploring PPD, in the Pakistani context, have not yet considered these factors which are much more relevant to cultural dynamics. The study concludes that the gender of the first-born child, social support, and family system matters most when considering the risk of PPD of firstborn infants' mothers.

\section{CONFLICT OF INTEREST}

There was no conflict of interest to be declared by any author. 


\section{REFERENCES}

1. O'Hara MW, McCabe JE. Postpartum depression: current status and future directions. Annu Rev Clin Psychol 2013; 9(1): 379-407.

2. Slomian J, Honvo G, Emonts P, Reginster JY, Bruyère O. Consequences of maternal postpartum depression: A systematic review of maternal and infant outcomes. Womens Health (Lond) 2019; 15: 1745506519844044.

3. Le Donne M, Mento C, Settineri S, Antonelli A, Benvenga S. Postpartum mood disorders and thyroid autoimmunity. Front Endocrinol 2017; 8(1): 91.

4. Lee YJ, Yi SW, Ju DH, Lee SS, Sohn WS, Kim IJ. Correlation between postpartum depression and premenstrual dysphoric disorder: Single center study. Obstet Gynecol Sci 2015; 58(5): 353-58.

5. Chen L, Wang X, Ding Q, Shan N, Qi H. Development of postpartum depression in pregnant women with preeclampsia: A retrospective study. Bio Med Res Intl 2019; 2019: 9601476.

6. Hahn-Holbrook J, Haselton M. Is postpartum depression a disease of modern civilization? Curr Dir Psychol Sci 2014; 23(6): 395-400.

7. Benoit C, Westfall R, Treloar AEB, Phillips R, Jansson SM. Social factors linked to postpartum depression: A mixed-methods longitudinal study. J Mental Health 2007; 16(6): 719-30.

8. Hahn-Holbrook J, Cornwell-Hinrichs T, Anaya I. Economic and health predictors of national postpartum depression prevalence: a systematic review, meta-analysis, and meta-regression of 291 studies from 56 countries. Front Psychiatry 2017; 8(1): 248-50.

9. Ali NS, Ali BS, Azam IS. Post partum anxiety and depression in peri-urban communities of Karachi, Pakistan: a quasi-experimental study. BMC Public Health 2009; 9(1): 384.

10. Tarar A, Mushtaq R. Postpartum depression: Prevalence and duration [B.Sc. Hons. Dissertation 2011]. GC University, Lahore, Pakistan; 2011.

11. Edinburgh postnatal depression scale (EPDS); 1987. Available from: URL: http://www.bbmomsclub.com/uploads/1/0/2/9/ 10293797/edinburghscale._eng-sp.pdf.

12. Kocalevent RD, Berg L, Beutel ME, Hinz A, Zenger M, Härter M, et al. Social support in the general population: standardization of the Oslo social support scale (OSSS-3). BMC Psychol 2018; 6(1): 31.

13. Ye Z, Wang L, Yang T, Chen LZ, Wang T, Chen L, et al. Gender of infant and risk of postpartum depression: a meta-analysis based on cohort and case-control studies. J Matern Fetal Neonatal Med 2020; 1(1): 1-10.

14. Anjum F, Akram M, Shaharyar R, Yaseen M, Batool Z, Zafar A. Assessing the role of maternal health care knowledge and practices in postpartum depression. J Soc Sci Res 2020; 6(9): 811-17.

15. Li Q, Yang S, Xie M, Wu X, Huang L, Ruan W, et al. Impact of some social and clinical factors on the development of postpartum depression in Chinese women. BMC Pregnancy Childbirth 2020; 20(1): 226-29.

16. Shewangzaw A, Tadesse B, Ashani T, Misgana T, Shewasinad S. Prevalence of postpartum depression and associated factors among postnatal women attending at hiwotfana specialized university hospital, Harar, East Ethiopia, 2015/2016. Open Acc J Repro Sexual Disord 2018; 1(1): 1-3. Available from: https:// lupinepublishers.com/reproductive-medicinejournal/ fulltext/ prevalence-of-postpartum-depression-andassociated-factors-among-postnatal-women-attending-at-hiwotfana-specialized-university.ID.000102.php.

17. Anokye R, Acheampong E, Budu-Ainooson A, Obeng EI. Prevalence of postpartum depression and interventions utilized for its management. Ann Gen Psychiatry 2018; 17(1): 18.

18. Shazad R, Yahya NM, Asima F. Postpartum Depression (PPD) among working and nonworking mothers/women in Karachi, Pakistan. J Nurs 2016; 6(3): 5-16.

19. Shah S, Lonergan B. Frequency of postpartum depression and its association with breastfeeding: A cross-sectional survey at immunization clinics in Islamabad, Pakistan. J Pak Med Assoc 2017; 67(8): 1151-56. 